\title{
Theoretical Study of the Effect of an AlGaAs Double Heterostructure on Metal-Semiconductor-Metal Photodetector Performance
}

\author{
Ali F. Salem, Student Member, IEEE, Arlynn W. Smith, and Kevin F. Brennan, Senior Member, IEEE
}

\begin{abstract}
The impulse and equare-wave input response of difierent GaAs metol-semiconductor-metal photodetector (MSM) designs are theoretically examined using a two dimenslonal drifdifrusion aumerical cajculation with a thermionic-field emiagion boundary condition model for the heterojunctions. The rise time and the fall time of the output signal current are calculated for a dmple GaAs, epitaxially erown, MSM device as well as for varlous double-heterostructure barrier devlces. The double heterostructure devices consist of an AJGaAs layer andwiched between the top Gass active, absorption layer and the bottom GaAs zubstrate. The effect of the depth of the AlGaAs layer on the speed and responsivity of the MSM devices is eramined. It is found that there is an optimal depth, at fixed applied bias, of the AIGaAs layer within the structure that provides maximum responsivity at minimal compromise in speed.
\end{abstract}

\section{INTRODUCTION}

M ETAL-semiconductor-metal (MSM) photodetectors are becoming increasingly attractive in optoelectronic communication systems, high-speed chip-to-chip connections, and high-speed sampling applications [1]. The principal advantages of MSM photodetectors that make them an excellent choice for on-chip detectors are their responsivity-bandwidth performance, compatibility with existing planar integrated circuit technologies, and relatively low voltage operation [1]. Improvement in MSM detector design structures, specifically the introduction of a GaAs/AlGaAs heterostructure, has advanced the realization of these applications [2].

The basic MSM detector structure is shown in Fig. 1. This structure consists of interdigitated metal fingers formed on the top surface of a semiconductor layer. Light incident on the top surface of the MSM structure is absorbed within the underlying semiconductor resulting in the creation of electron-hole-pairs (EHP's). The application of a bias to the metallic fingers creates an electric field within the underlying semiconductor which acts to sweep the photogenerated carriers out of the device. How fast these carriers are collected and how many of them actually survive to the contacts within a particular collection time determine the speed and the responsivity,

\footnotetext{
Mnnuscrip received October 6, 1993; revined Februery 22, 1994. The review of this paper was arranged by Associate Editor P. K. Bhanacharya. This work was supported in parn by Digital Equipment Corp. through Contract E21-H36, in part by the National Science Foundation through a Pretidentid Young Investigator Award made to K. Brennan, and in part by ARPA through NASA Contract NAGW-2753.

The authors are with the School of Electrical and Computer Engineering. and Microelectronics Research Center, Georgia Institule of Technology. Allents. GA 30332-0250 USA.

IEEE Log Number 9401527.
}

\section{CENEUA MIAL-SEMCONDUCTOR-MTAN GTLUTUL}

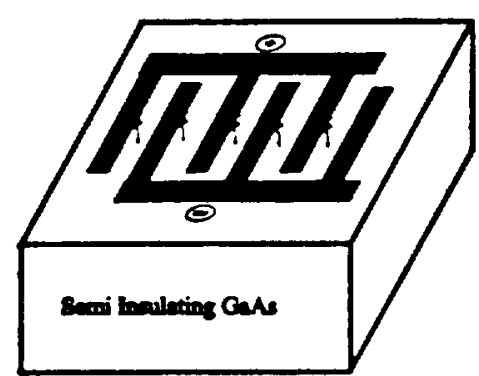

Fig. 1. Schematic diagram of an interdigitaled mets-eemiconductor-metal (MSM) structure.

respectively, of the detector. Carriers generated deep within the semiconductor must traverse a greater distance before they are collected at the contacts compared to those generated near the surface. Depending upon the magnitude of the electric field within the semiconductor, the time needed to collect those carriers generated deep in the device can vary drastically. Under low bias conditions, magnitude of $5-10 \mathrm{~V}$, which is typical for most integrated circuit applications, this collection time can be prohibitively long in high speed applications.

It has been suggested that the insertion of a double heterostructure layer can improve the time response of MSM detectors [2]. The double heterostructure layer acts to block those carriers generated deep within the device structure. As a result, only those carriers photogenerated within the top absorption layer are collected leading to a fast overall response. However, as described above, the responsivity principally depends upon the number of photogenerated carriers collected at the contacts. A high responsivity, especially at low input power levels, dictates that most of the photogenerated carriers be collected. Since many of the photogenerated carriers are produced deep within the semiconductor layer, the insertion of a double heterostructure layer in order 10 improve the speed of the device, necessarily reduces its responsitivity as well. Therefore, there exists a fundamental tradeoff between the speed of response and responsivity of a heterostructure MSM detector.

It should be noted that the absorption coefficient varies strongly with the optical wavelength. In this paper, we examine the response of the photodetector to only one incident wavelength, $840 \mathrm{~nm}$. The speed of response of the detector can 
be quite different at other wavelengths since the absorption may occur closer to the surface or deeper into the device. If for example, the absorption depth is much smaller than the interdigitated finger spacing then the detector's speed of response is limited by the finger separation and the placement of the AIGaAs layer has minimal effect. However, at an incident wavelength of $840 \mathrm{~nm}$ the absorption depth and finger separation are comparable and the placement of the AJGaAs layer becomes important. Therefore, the tradeoff between the speed of response and the responsitivity discussed herein applies principally to incident light at $840 \mathrm{~nm}$.

In this paper, we present results of a theoretical study of the performance of a GaAs based MSM detector examining the effect of including a sandwiched AlGaAs layer between the active GaAs layer and the substrate. A two-dimensional drifi-diffusion numerical calculation in combination with a thermionic-field-emission model is employed in this study. The drifi-diffusion/thermionic emission model along with the details of the numerical solution method used are described in Section II. Calibration of the model and comparison to existing models is discussed in Section III. In Section IV different GaAs MSM double heterostructure devices are examined using the model. In Section IV we examine the effect of the AlGaAs layer on the rise time, fall time, and the magnitude of the output signal current and optimize the placement of such a layer as it relates to these parameters.

\section{MODEL DESCRIPTION}

To understand the behavior of photo-generated carriers under the influence of drift and diffusion forces, Poisson's equation, the current continuity equations for electron and holes, and a rate equation for charged traps are solved on a two dimensional simulation domain for the electrostatic potential, and the carrier concentrations. The drift-diffusion approach is sufficient to characterize the operation of MSM detectors since these devices are typically of the order of microns in length and width. In this study, the detectors examined are restricted in dimensions to several microns where the driftdiffusion approximation is valid. The basic equations used in the simulator are [3], [4]:

$$
\begin{aligned}
\epsilon_{0} \epsilon_{0} \nabla^{2} \psi & =-q\left(p-n+N_{D}-N_{A}+n_{t}\right) \\
-\frac{1}{q} & \nabla \cdot J_{n}-G_{n}+R_{n}+\frac{\partial n}{\partial t}=0 \\
\frac{1}{q} & \nabla \cdot J_{p}-G_{p}+R_{p}+\frac{\partial p}{\partial t}=0 \\
J_{n} & =q \mu_{n} n E_{n}+q D_{n} \nabla n \\
J_{p} & =q \mu_{p} p E_{p}-q D_{p} \nabla p \\
\frac{d n_{t}}{d t} & =\left(R_{n}-G_{n}\right)_{S R H}-\left(R_{p}-G_{p}\right)_{S R H}
\end{aligned}
$$

where $\Psi$ is the electrostatic potential, $n$ and $p$ are the electron and hole carrier concentrations, $N_{D, A}$ are the donor/acceptor concentrations, $n_{t}$ is the electron-filled trap concentration, $J_{n, p}$ are the electron/hole currents, $R_{n, p}$ and $G_{n, p}$ are the electron/hoje recombination and generation rates, $\mu_{n, p}$ and $D_{n, p}$ are electron/hole mobilities and diffusivities, and the subscript SRH in (6) denotes the Shockley-Read-Hall events.
In most situations, the trap density is such that the effects of (6) are negligible [3]. The terms $R_{n, p}$ used in (2) and (3) represent the sum of Shockley-Read-Hall, radiative, and Auger recombinations for electrons and boles [4]. The optical generation rate, $G$, is generally assumed to be gaussian for the impulse response and is typically given as [5]

$$
\begin{aligned}
G_{n}= & G_{p}=\frac{W_{p}}{h v} \alpha_{\text {abs }} \cdot \exp \left(-\frac{\left(t-t_{0}\right)^{2}}{\sigma_{t}^{2}}\right) \\
& \exp \left(-\frac{\left(x-x_{0}\right)^{2}}{\sigma_{x}^{2}}\right) \cdot \exp \left(-\alpha_{\text {aba }} y\right)
\end{aligned}
$$

where $W_{p}$ is the peak optical power density incident on the surface, $h v$ is the photon energy, $\alpha_{a b}$ is the absorption coefficient, $x_{0}$ is the location of the center of the beam, $t_{0}$ is the location of the optical input peak, $\sigma_{t}$ and $\sigma_{2}$ are related to the full width, half maximum (FWHM) of the pulse. In this paper, we examine the response of only a representative unit cell of the device in which the illumination is practically uniform. The beam is assumed to be centered within the unit cell. Given the dimensions of the unit cell, the lateral decay of the beam is negligible within the calculations presented here. Therefore. for simplicity, uniform illumination is assumed laterally within the unit cell and the spatial exponential term in (7) is set to unity. The metallic fingers are assumed to be completely transparent. In practice, there are of course shadowing and reflective losses at the metallic surface which would lead to a reduction in the amount of photogenerated carriers within the underlying semiconductor material. For simplicity and due to a lack of detailed information about the extent of these losses, we assume here that no losses occur.

A standard field-dependent mobility for the electron mobility, $\mu_{n}[4]$ is used,

$$
\mu_{n}=\frac{\mu_{n o}+v_{0} \frac{F^{3}}{F_{0}^{4}}}{1+\left(\frac{F}{F_{0}}\right)^{4}}
$$

where $\mu_{n o}$ is the zero field mobility, $F_{0}$ is the critical electric field, $F$ is the local electric field, and $v_{b}$ is the saturation velocity. The hole mobility is assumed to be constant, independent of the electric field. It is further assumed that the Einstein relation holds for the diffusivities. The use of the Einstein relation, though universally accepted, is not totally correct since it applies only strictly to equilibrium. Improvement over the Einstein relation necessitates determining the mobility and diffusivity using more exact methods of solution of the Boltzmann equation, i.e., the ensemble Monte Carlo technique. Presently, this information is not available to us and thus for simplicity, the Einstein relation is adopied as is typically done in drifi-diffusion solutions.

The model's modifications made to handle heterojunctions follow the same approach as given by Sutherland and Hauser [6]. In addition, a thermionic-field emission boundary condition is used to specify the current density at heterojunction interfaces. The thermionic emission boundary condition [7], [8] is implemented in parallel with the drift-diffusion model. The actual current actoss the heterointerface is limited by 
either the themionic emission or diffusion current, depending upon which is smaller. In the present model, the drifi-diffusion and thermionic emission current densities at the heterointerface are computed and compared. The actual current density across the heterointerface is then given by the smaller of these two quantities ensuring that the current across the junction is either thermionic emission or diffusion limited. Tunneling across the heterojunction is modeled based on the theory of Crowell and Rideout [9] and as developed in [10]. The tunneling current density is added at the heterojunction interface to give the total current density across the interface.

Equations (1)-6) along with the proper boundary conditions can be solved for the three fundamental variables $\Psi, n$, and $p$. The Dirichlet boundary conditions of the electrostatic potential used at the Schottky contacts are

$$
\psi=\psi_{b}+\psi_{\mathrm{app}}-\psi_{0}
$$

where $\Psi_{b}$ is the built-in potential, $\Psi_{\text {app }}$ is the applied bias voltage, and $\Psi_{\text {, }}$ is the Schottky barrier height. From the thermionic emission and diffusion theory of Crowell and Sze [11] the carrier concentrations at the Schotky contacts are specified in terms of the current density passing through them as [3], [4], [12]:

$$
\begin{aligned}
& J_{n} \cdot \hat{n}=-q v_{n}\left(n-n_{o}\right) \\
& J_{p} \cdot \hat{n}=q v_{p}\left(p-p_{0}\right)
\end{aligned}
$$

where $v_{n, p}$ are the electron/hole thermionic recombination velocities, $\hat{n}$ is the unit normal vector, and $n_{0}$ and $p_{0}$ are the equilibrium electron and hole carrier recombination at the Schotky contacts. Specifically, $n_{0}$ and $p_{0}$ are given as

$$
\begin{aligned}
& n_{o}=N_{c} e^{\left(-q \psi / k_{B} T\right)} \\
& p_{o}=N_{v} e^{\left(q\left(\psi-E_{\text {aop }}\right) / k_{B} T\right)}
\end{aligned}
$$

where $N_{c}$ and $N_{v}$ are the electron and hole effective density of states, and $E_{\text {gap }}$ is the energy gap. At any interface, Gauss's law can be applied to relate the normal component of the electric flux density to the interface charge as

$$
\left.\varepsilon_{\text {mat1 }} \cdot \frac{\partial \psi}{\partial n}\right|_{\text {mat1 }}-\left.\varepsilon_{\text {mat2 }} \cdot \frac{\partial \psi}{\partial n}\right|_{\text {mat2 }}=Q_{\mathrm{INT}}
$$

where $Q_{\text {int }}$ is the interface charge. The free surface carrier concentrations are determined from conditions on the normal current [4], [12]:

$$
\begin{aligned}
& J_{n} \cdot \hat{n}=q \cdot R^{\text {eurf }} \\
& J_{p} \cdot \hat{n}=q \cdot R^{\text {surf }}
\end{aligned}
$$

where $R^{\text {eurf }}$ is the surface recombination rate. Equations (12)-(14) reduce to the usual Neumann boundary conditions when the interface charge and the surface recombination rates vanish.

The box integration approach of the finite difference method [4] is used to discretize (1)-(6). These equations are then solved with their appropriate boundary conditions on a nonuniform, two-dimensional mesh consisting generally of $75 \times 33$ points. The Scharfetter-Gummel technique [4], [13] is applied in the formulation of the discretization equations. The resulting system of equations is linearized using Newton's method. Due to storage and computation time limitations the Gaussian elimination method is deemed inappropriate for the solution of the linearized system. Therefore, the system of equations is solved using an iterative approach known as the bi-conjugate gradient squared (BICGS) method [14], [15]. Only nonzero elements in the matrix are stored using this approach greatly reducing the data storage requirements of the code. After the program reaches convergence, the potential and carrier concentration profiles are readily available, from which most of the macroscopic variables of interest can be calculated. The current densities, $J_{n}$ and $J_{p}$, are calculated from (4) and (5) using Scharfetter-Gummel's exponential scheme [13] for the carrier concentrations.

\section{Calibration of the Model}

To demonstrate the accuracy of the model described above it may seem at first that direct comparison to experimental measurements would be best. However, this is not an easy task since information about the experimental setup is actually needed to accurately compare the theoretical results to experimental measurements. Different authors [5], [16] have attempted comparing the calculated response of GaAs MSM photodetectors from the drift diffusion model to experimental measurements. Landheer et al. [16] found that an equivalent circuit model for the experimental setup is needed to accurately compare the theoretical results to experimental measurements. The output current predicted by the circuit model of Landheer et al. [16] did not lead to very accurate agreement with the experimental measurements. This discrepancy is apparently due to the simplistic treatment of the extemal circuit and the lack of complete knowledge of the experimental circuit parameters. Alternatively, Sano [5] proposed an analytical model for GaAs MSM photodetectors based on the solution of the drift diffusion equations (1) (6). The analytical model used is an equivalent RC circuit model in which the resistance, $R$, and the capacitance, $C$, are calculated based on the electric field and carrier concentrations obtained from the drift diffusion solution. The equivalent circuit model was implemented in a SPICE-like circuit simulator and the transient responses from the circuit simulation were compared with measured responses for an MSM photodetector. Though Sano [5] achieved good agreement between the circuit simulator and the experimental measurements, this is accomplished by numerically adjusting the circuit parameters in the model to ensure agreement with the experiment. Therefore, direct comparison of the drift diffusion results to experiment is hampered by the insufficient information about the experimental setup used in the measurements. In the absence of information about the experimental setup we choose to compare our model instead to other existing numerical models.

We have compared the results obtained using the present model against several existing device simulators and against a 1-D analytical solution for a simple $1 \mu \mathrm{m}$ GaAs $p-n$ junction diode. The donor and acceptor concentrations chosen for this test device are $N_{D}=N_{A}=10^{17} \mathrm{~cm}^{-3}$ and the carrier mobilities are $\mu_{n}=7000 \mathrm{~cm}^{2} / \mathrm{V}$-s and $\mu_{p}=300 \mathrm{~cm}^{2} / \mathrm{V}$-s. 


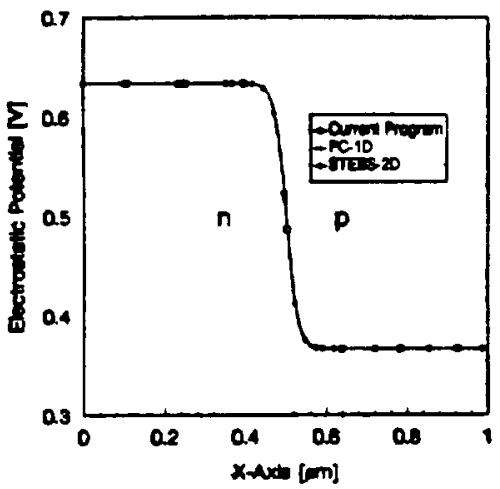

Fig. 2. Comparison of the electrosutic polentials obtained from three different numerical solutions for a GaAs pn junction diode forward biesed at $1 \mathrm{~V}$ : current program (circles), PC-1D (diamonds), and STEBS-2D (equares).

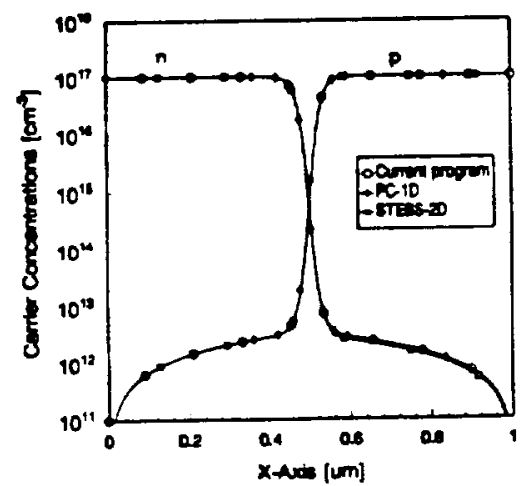

Fig. 3. Comparison of the electron and hole concentrations for a GaAs pn junction diode forward biased at I $\mathrm{V}$ calculated from the current program (circles), PC-1D (diamonds), and STEBS-2D (squares).

Fig. 2 shows the calculated electrostatic potential $\Psi$ obtained from the present model and from two other simulators, PC-1D [17] and STEBS-2D [18]. PC-1D is a commercial, onedimensional, drift-diffusion model released by Sandia National Laboratory and STEBS-2D is a full hydrodynamic model developed at the Georgia Institute of Technology. The carrier concentrations obtained from the three models are also presented in Fig. 3. As can be seen from inspection of Figs. 2 and 3, the present program shows precise agreement with the other two models under comparable conditions.

The electrostatic potential within the p-n junction diode calculated using the present model can also be compared to a simple one-dimensional analytical solution of Poisson's equation using the depletion approximation. Inspection of Fig. 4 shows good agreement between the numerical model and the analytical model except near the edge of the depletion region where the depletion approximation fails to properly account for the tails in the carrier distributions.

\section{APPLICATION TO MSM PHOTODETECTORS}

The present numerical model is applied to study the response of GaAs based metal-semiconductor-metal (MSM) photodetectors. The general device structure considered is sketched in Fig. I above. The barrier height assumed for the Schortky contacts in the structure is $0.7 \mathrm{~V}$ and the GaAs material is taken as semi-insulating with a background doping concentration of $N_{D}=10^{15} \mathrm{~cm}^{-3}$. The interdigitated

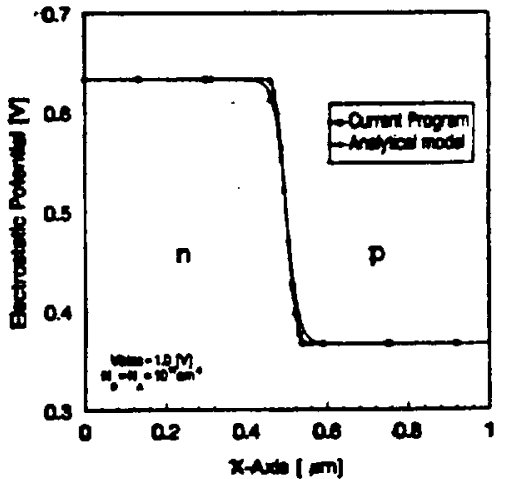

Fig. 4. Comparison of the electrostatic potentials for $:$ GaAs pn junction diode forward-biased at 1 V calculated from the present model (circles) and a one-dimensional analytical colution (diamonds) using the depletion eproximation.

TABLE I

Materul Parangters Used

\begin{tabular}{|c|c|c|c|c|c|}
\hline Parameter & Units & GaAs & Ref. & AGaAs & Ref \\
\hline Electron Mass ( $\left.m_{n}^{*}\right)$ & - & 0.061 & [18] & 0.088 & [18] \\
\hline Hole Mass ( $\left.m_{p}^{*}\right)$ & 一 & 0.48 & [18] & 0.56 & [18] \\
\hline Dielectric Constant $(\varepsilon)$ & - & 13.1 & [18] & 12.4 & [18] \\
\hline Energy $\operatorname{Gap}\left(E_{\rho}\right)$ & (eV) & 1.42 & [18] & 1.74 & [18] \\
\hline Electron Affinity $(x)$ & (eV) & 4.06 & [19] & 3.80 & [19] \\
\hline$y\left(\mu_{n}\right)$ & $\left(\mathrm{cm}^{2} / \mathrm{Vs}\right)$ & 7000 & [19] & 2500 & [19] \\
\hline Hole Mobility $\left(\mu_{p}\right)$ & $\left(\mathrm{cm}^{2} / \mathrm{Vs}_{\mathrm{s}}\right)$ & 300 & [19] & 150 & [19] \\
\hline ime $\left(\tau_{n}\right)$ & (s) & $10^{-7}$ & [20] & $10^{-8}$ & [19] \\
\hline Hole Lifetime $\left(\tau_{p}\right)$ & (s) & $10^{-7}$ & [20] & $10^{-8}$ & [19] \\
\hline Radis & $\left(\mathrm{cm}^{3} / \mathrm{s}\right)$ & $2.04 \times 10^{-10}$ & [21] & $2.04 \times 10^{-10}$ & 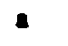 \\
\hline $\begin{array}{l}\text { Electron Auger Coef } \\
\left(C_{n}\right)\end{array}$ & $\left(\mathrm{cm}^{6} / \mathrm{s}\right)$ & $16 \div 10-29$ & [21] & $1.6 \times 10^{-30}$ & a \\
\hline Hole Auger Coef. ( $C$ & & $.64 \times 10$ & {$[21]$} & $1.64 \times 10^{-}$ & 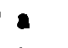 \\
\hline Absorption Coef. & $\left(\mathrm{cm}^{-1}\right)$ & $10^{4}$ & [22] & 0 & b \\
\hline
\end{tabular}

Due to lack of information for AGaAs. GaAs values are used.

-No absorption takes place for the AJGaAs a $\lambda=0.84 \mu \mathrm{m}$.

TABLE II

INPUT SIGNAL PARAMETERS

\begin{tabular}{ll}
\hline Peak Power $\left(W_{p}\right)$ & $0.68 \mathrm{~mW} / \mathrm{cm}^{2}$ \\
Wavelength $(\lambda)$ & $840 \mathrm{~mm}$ \\
Beam Diameter $(D)$ & $60 \mu \mathrm{m}$ \\
Beam's Peak Position $\left(X_{0}\right)$ & $2.5 \mu \mathrm{m}$ \\
Signal Peak $\left(t_{0}\right)$ & $10 \mathrm{ps}$ \\
Full Width Half Max. (FWHM) & $5 \mathrm{ps}$ \\
\hline
\end{tabular}

metallic finger widths and spacings are $1 \mu \mathrm{m}$ and $3 \mu \mathrm{m}$, respectively. The material parameters used in the calculation, i.e., the zero field carrier mobilities, lifetimes, etc. are compiled in Table I. These parameters are compiled from [19]-[23]. The parameters used to characterize the optical input signal are collected in Table II. In these calculations the boundary condition at the bottom surface and along the sides of the device is assumed to be floating.

The response of the GaAs MSM device as sketched in Fig. 1 is compared to a double heterostructure device. The double heterostructure device consists of an AlGaAs barrier layer of thickness $d_{2}$, sandwiched between two different GaAs layers, of thicknesses $d_{1}$ and $d_{3}$, as shown in Fig. 5. The Al concentration within the AIGaAs layer is assumed to be 


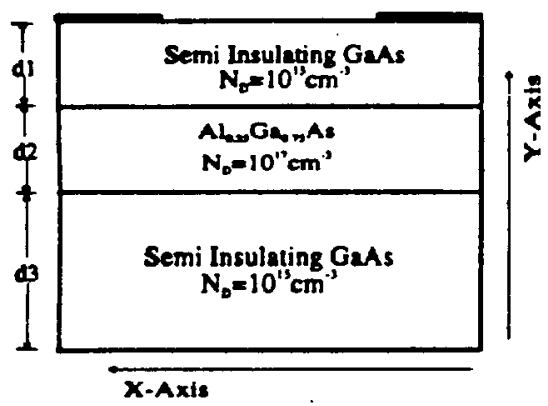

Fis. 5. Schemetic diagram of the double-barriet heterostructure MSM showing the sandwiched AGaAs layer. The solid shaded regions on the lop of the figure represent the Schorky contacts.

held fixed at 25\%. Different double heterostructure devices are examined with variable depths of the AlGaAs barrier layer. As a result, the active, photo-absorption layer thickness $d_{1}$, obviously also varies in these devices.

As described above, the primary function of the buried heterostructure layers is to block the collection of the carriers photogenerated deep within the GaAs substrate by preventing them from diffusing back into the active layer and towards the contacts. In addition, at low applied bias, the barrier between the active and the AlGaAs layers acts to confine the photogenerated carriers within the active region. At higher applied bias, the heterostructure blockage of the photogenerated carriers becomes less effective due to the much greater band bending present in the device. Subsequently, the location of the AJGaAs layer greatly effects the charge collection attributes of the device depending upon the field distribution and the applied bias. In the structures examined here, the background doping is n-type, implying that the primary photogenerated carriers collected are holes.

The calculated impulse response at different active layer thicknesses, along with the corresponding rise and fall times of the signal are displayed in Fig. 6. Five different structures are examined, four double heterostructure devices and one GaAs bulk device $6 \mu \mathrm{m}$ in thickness. The double heterostructure devices consist of top GaAs layer, $d_{1}$, ranging in thickness from 0.5 to $4 \mu \mathrm{m}$, a $1 \mu \mathrm{m} \mathrm{AlGaAs} \mathrm{layer,} d_{2}$, followed by a Gas epilayer, $d_{3}$, ranging in thickness from 4.5 to $1 \mu \mathrm{m}$. Notice that the total width of all three layers combined remains constant at $6 \mu \mathrm{m}$. The rise and fall times are defined as the time it takes the output signal to go from $10 \%$ to $90 \%$ and from $90 \%$ to $10 \%$ of its maximum value, respectively. The voltage applied to the device is $-5 \mathrm{~V}$. As can be seen from Fig. 6, the fastest response occurs for the device configuration with a $0.5 \mu \mathrm{m}$ active layer thickness. This is obvious from both the curve corresponding to the $0.5 \mu \mathrm{m}$ device as well as from its corresponding fall time. However, the maximum output signal magnitude for the $0.5 \mu \mathrm{m}$ device is significantly less than for the other cases. This is as expected, since the response speed is achieved at the expense of lower output signal magnitude because the slower carriers, those generated deep within the device, are blocked from being collected by the heterojunction barrier. As the active layer thickness $d_{1}$ increases to $1 \mu \mathrm{m}$, more carriers are generated within the top, active GaAs region. As a result, greater number of

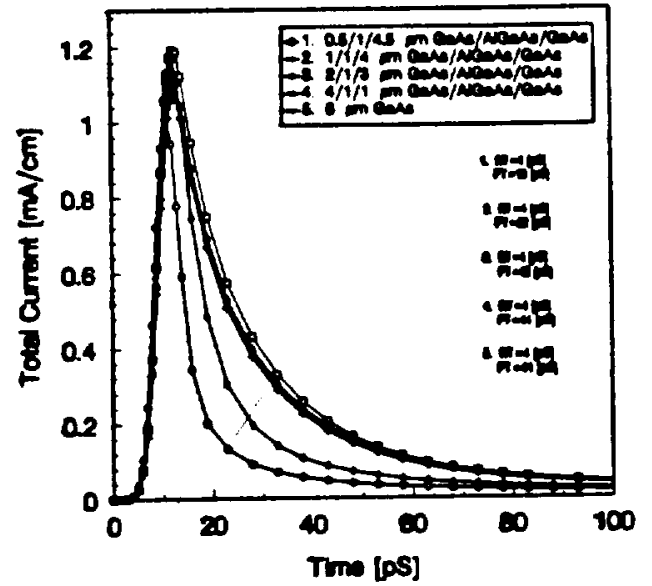

Fig. 6. The calculated impulse response at different extive layer thickneases for the double-berrier MSM heterostricture at an applied bias of $-5 \mathrm{~V}$. The doping levels are $10^{15} \mathrm{~cm}^{-3}$ for the GaAs hyen and $10^{17} \mathrm{~cm}^{-2}$ for the AGaAs layer. Curve 5 is for the case withoul the AGaAs layer. The numbers in the legend repreaent the layer thickness in microns of the GaAs/AJGaAs/GaAs layers as shown in Fig. S. Aso included are the rise and fall times for each of the 5 curves.

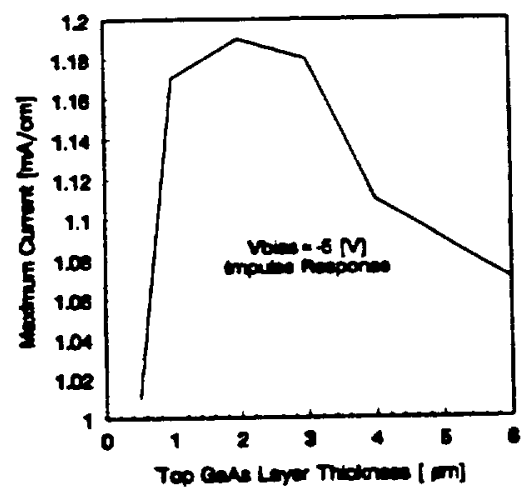

Fig. 7. The maximum output current signal as a function of ective layer thickness for the double-barrier MSM beterostructure.

photogenerated carriers are collected producing a higher output signal current. Though the $d_{1}=1 \mu \mathrm{m}$ device does not show as rapid a collection of the photogenerated carriers as the $d_{1}=0.5 \mu \mathrm{m}$ device, the field is sufficiently strong and the carriers are still relatively close to the collecting contacts that a reasonably high speed of response is retained; a fall time of $22 \mathrm{ps}$ is achieved as compared to $13 \mathrm{ps}$ for the $0.5 \mu \mathrm{m}$ device. If the active layer thickness is increased further to $2 \mu \mathrm{m}$, the same trend is observed; more carries are collected from the bottom of the active layer resulting in a longer fall time, $\sim 42$ ps, and slower speed of response. Interestingly, the maximum output signal current ultimately decreases with increasing $d_{1}$. The maximum signal current is plotred as a function of active layer thickness in Fig. 7. As can be seen from Fig. 7, the output signal current reaches a maximum for an active layer thickness of $d_{1}=2 \mu \mathrm{m}$. The maximum signal current is significantly less for a device with $d_{1}=4 \mu \mathrm{m}$ than with $d_{1}=2 \mu \mathrm{m}$, though the fall times are comparable. Clearly, at an applied bias of $-5 V$, there exists an optimal thickness, in terms of speed of response and collection efficiency, for layer $d_{1}$.

In an attempt to understand the origin of the peak in the output current signal versus active layer thickness curve, we 


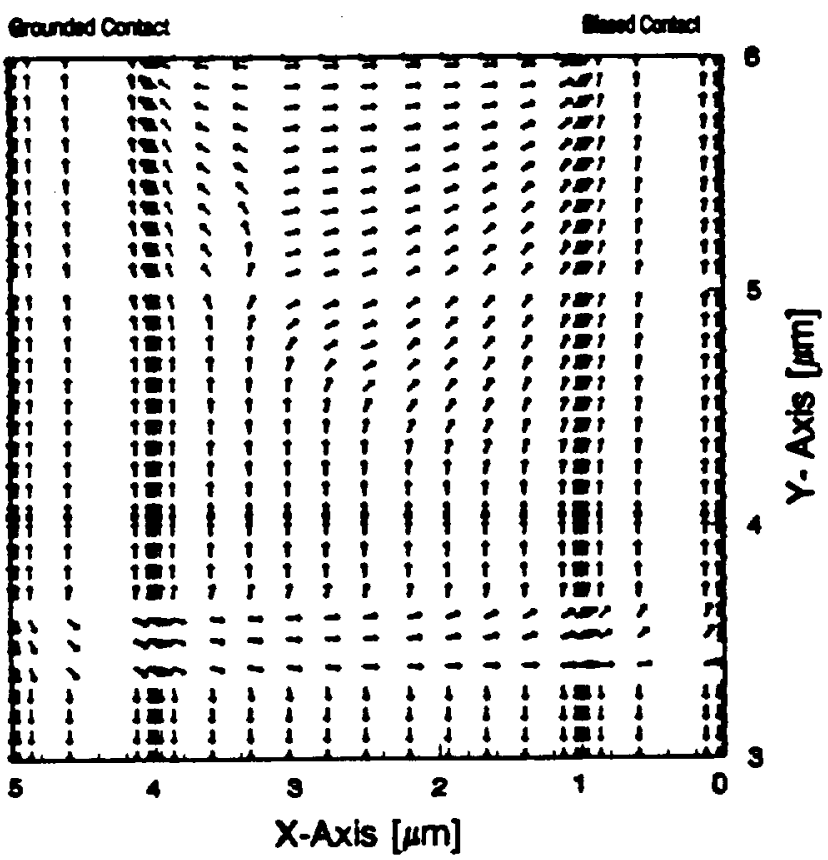

Fig. 8. Electric field vector plot at the time-step corresponding to the peak current showing the direction of the electric field at each mesh point within the two-dimensional simulation grid used for the $2.0 \mu \mathrm{m}$ active layer thickness device. The AGaAs layer is located $2.0 \mu \mathrm{m}$ from the top and is $1.0 \mu \mathrm{m}$ in thickness. Only the top $3 \mu \mathrm{m}$ of the device is shown in the figure. The right lop conuct of the device is biased at $-5 \mathrm{~V}$ with respect 20 the left top contsct. The horizontal channeling of the electric field at the middle of the heterobarrier is due to the accumulation of electrons within the AIGaAs trapped by the potential band bending at either interface. Notice that the lateral component of the electric field vanishes within the active region above the beterobarrier.

have examined the electric field profiles within each device. Field vector plots showing the direction of the electric field at each mesh point within the device are shown in Figs. 8 and 9 for the 2.0 and $4.0 \mu \mathrm{m}$ active layer thickness devices, respectively. Comparison of the two figures clearly shows that the lateral field component essentially vanishes near the heterostructure in the $2.0 \mu \mathrm{m}$ width device, while a significant lateral component persists within the $4.0 \mu \mathrm{m}$ width device. The electric field points vertically away from the heterostructure acting to accelerate the photogenerated holes towards the collecting contacts in the $2.0 \mu \mathrm{m}$ device. Altematively, in the $4.0 \mu \mathrm{m}$ device, the lateral component of the electric field acts to acclerate the holes to some extent laterally. As a result, the hole trajectory for collection is longer, effectively delaying their collection at the contacts. Since the holes spend more time in this region their chances of suffering a recombination event increase, leading to a reduction in the collected current. Subsequently, this could result in an increased collected current within the $2.0 \mu \mathrm{m}$ width device than in the $4.0 \mu \mathrm{m}$ width structure. A more detailed analysis of this problem using a more sophisticated, hydrodynamic model will be made in the future to further test this hypothesis.

The effect of the heterojunction on the response to a square wave input is considered next. The same model and approach as described above is used but with square wave input signal applied to the device. The output current response to a square wave input with repetition rate of $1 \mathrm{GHz}$ and $0.5 \mathrm{~ns}$ duration time is shown in Fig. 10 for different GaAs active

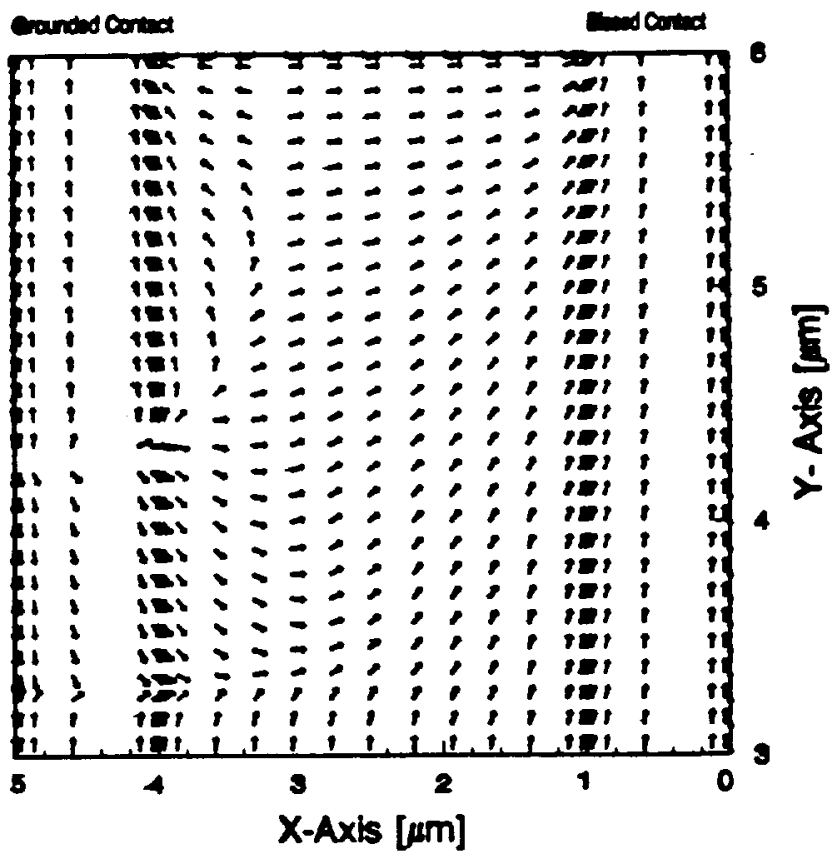

Fig. 9. Electric field vector plot at the time-step corresponding to the pesk current showing the direction of the electric field al each mesh point within the two-dimensional simulation grid used for the $4.0 \mu \mathrm{m}$ ective layer thickness device. The NGaAs layer is localed at $4.0 \mu \mathrm{m}$ from the lop and is 1.0 $\mu \mathrm{m}$ in thickness. Only the top $3 \mu \mathrm{m}$ of the device is shown in the figure. The right top contact of the device is biased at $-5 \mathrm{~V}$ with respect to the left top contact. Notice that the electric field "swirls" around deeper within the setive region of the device. The presence of the lateral component of the electric field acts to increase the trajectory of the carriers leading to a longer time between generation and collection with the subsequent increase of recombination losses.

layer thicknesses. The applied voltage is again $-5 \mathrm{~V}$. It is clear from this figure that the output signal amplitude peaks at an active layer thickness of $2 \mu \mathrm{m}$ and then decreases as the active layer gets thicker. The explanation for this is similar to that described above for the impulse response. The rise and fall times however are not quite consistent with the impulse response results. This is due primarily to the definition of these quantities. Although the $0.5 \mu \mathrm{m}$ curve in Fig. 10 seems to decay faster than any of the other cases, the fall time, for example, is not the shortest. This is due to the fact that the 90-10\% measure of the fall time depends on the magnitude of the signal peak itself. Since the signal peak is very much smaller in the $0.5 \mu \mathrm{m}$ device, its decay to $10 \%$ takes longer than that for a much higher signal peak, though the signal in the $0.5 \mu \mathrm{m}$ device has decayed quicker below some threshold level. Subsequently, the definition of the rise and fall times typically given, 10-90\% and 90-10\% respectively, are of questionable value when evaluating the performance of an MSM detector subject to a square wave input. However, it is clear that the presence and the location of the AlGaAs layer is critical to the performance of these photodetectors and that the optimal location of the heterojunction barriers is a function of the applied bias. This is clearly demonstrated in Fig. 11 where the applied voltage is increased to $-10 \mathrm{~V}$. We note that in this case the peak in the collected current shifts to the $3 \mu \mathrm{m}$ active layer thickness device. The shifi in the peak collected current occurs since the field depletes deeper into the semiconductor 


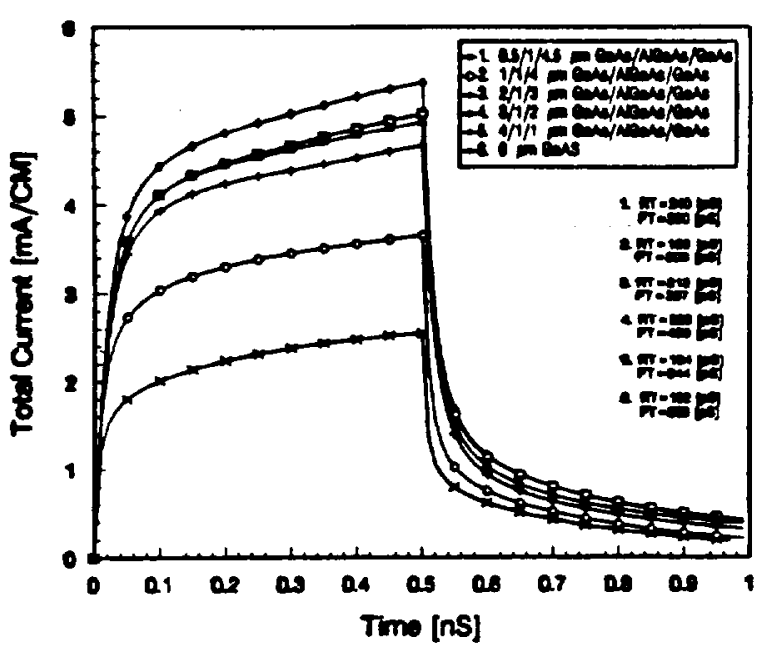

Fig. 10. The calculated outpul current response of the double-bartier MSM beterostructure to a square-wave input at different active layer thicknesues and under an applied voliage of $-5 \mathrm{~V}$. Curve 6 is for the case without the AlGaAs layer. The numbers in the legend represent the layer thickness in microns for the GaAs/AJGaAs/GaAs layers as ahown in Fig. S. Aloo included are the rise and fall times in picoseconds for each of the curves.

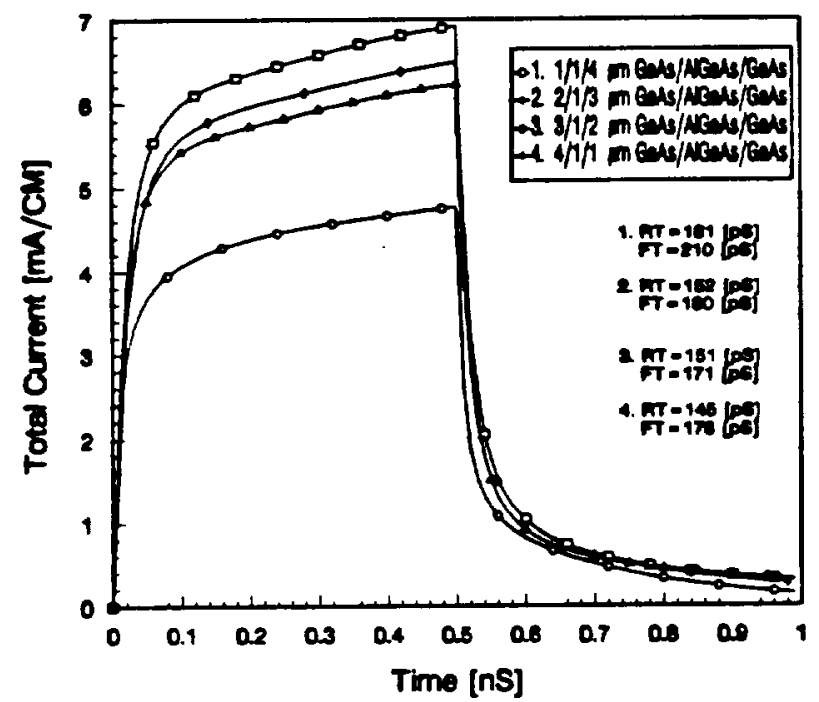

Fig. 11. The calculated output current response of the double-barrier MSM helerostructure to a square-wave input at different active layer thicknesses and under an applied bias of $-10 \mathrm{~V}$. Curve 6 is for the case without the AlGaAs layer. The numbers in the legend represent the layer thickness in microns of the GaAs/AJGaAs/GaAs layers as shown in Fig. 5. Also included are the rixe and fall times in picoseconds for each of the curves.

material. Subsequently, more carriers are swept out by the relatively high-field producing a greater collected current at the contacts.

Due to computational limitations, it is presently possible to simulate the entire substrate. Subsequently, only small portion of the substrate can be simulated. In this case, the bottom surface can be treated either as floating or as an ohmic contact. The use of an ohmic boundary condition along the bottom surface leads to qualitatively similar results as those with the floating surface condition but with some quantitative difference. The appropriateness of these two boundary conditions needs to be further investigated, which wil be reported in a future work.

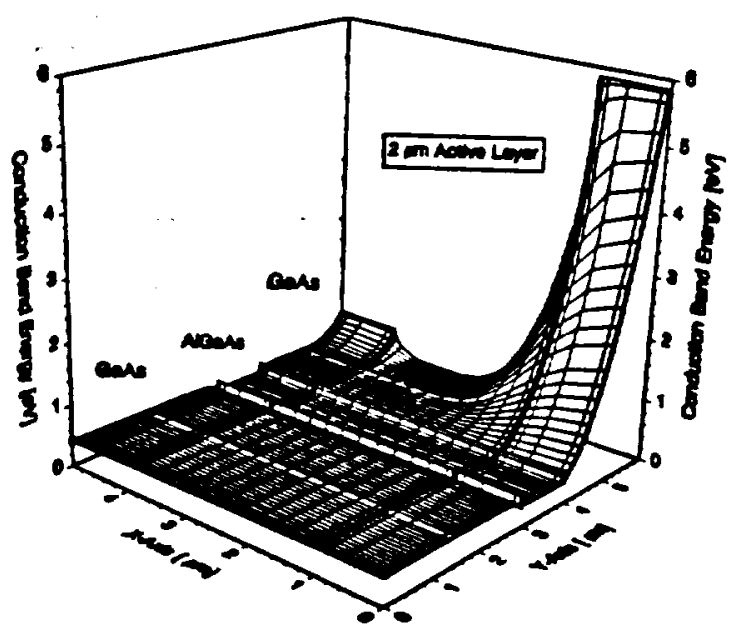

Fig. 12. Two-dimensional conduction bend diverum of the double-barier MSM heterostructure for the $2 \mu \mathrm{m}$ ective layer thicbness device at an applied bins of $-5 \mathrm{~V}$.

Finally, we examine the dark current for the device sketched in Fig. 1 assuming a finger length of $100 \mu \mathrm{m}$, typical for most MSM structures. The dark current is found to be on the order of $0.6 \mathrm{pA}$. Moreover, it is also found that it is practically insensitive to the applied bias in the range from zero to -20 $V$. This is because, at a $3 \mu \mathrm{m}$ finger spacing, and at the applied biases considered here, the built-in potential barrier at the grounded Schottky contact persists as shown in the two-dimensional conduction band plot for the $2.0 \mu \mathrm{m}$ active layer thickness device in Fig. 12. However, if the voltage is increased or if the finger spacing is reduced, the applied voltage at the biased contact punches through to the grounded contact reducing the built-in potential barrier. As a result, an increased electron dark current develops. It is expected then that for a smaller finger spacing $(\sim 1 \mu \mathrm{m})$ and a doping level of $10^{15} \mathrm{~cm}^{-3}$ that the dark current will increase with increasing applied voltage [24], [25]. In that case, a top AlGaAs layer [26] can play an important role in limiting the dark current of the device.

\section{CONCIUSions}

We have theoretically investigated the effect of including - double heterostructure barrier on the response of MSM intendigitated photodetectors using a two-dimensional drifdiffusion model with a thermionic-field emission boundary condition. To establish the validity of the model we have compared it to existing models and to a one-dimensional analytical solution. Excellent agreement with these models is obtained. The drift-diffusion model is then used to study the effect of the presence of a sandwiched AIGaAs layer on the response, as measured by the responsivity and speed, of MSM photodetectors. The AIGaAs layer introduces a double heterostructure barrier whose location in the structure greatly affects the movement of the carriers. We have found that there exists an optimal location for these heterojunction barriers for which both high speed and high responsivity can be achieved for an input optical signal of $840 \mathrm{~nm}$ wavelength. The placement of the AJGaAs barrier depends on the applications in which the MSM's are to be used. In high speed applications, 
and under low bias conditions, the AlGaAs layer should be placed near the top surface of the device. To obtain a fast response under low light illumination levels, the active layer thickness and the applied bias should be increased. However, operation at high bias can increase the dark current levels of the device if the electrode spacing is small. Subsequently, there exist several tradeoffs in the design of an interdigitated MSM photodetector. Use of a simulator such as the one described here, is essential for optimizing a structure for a particular application.

\section{ACKNOWLEDGMENT}

The authors would like to thank Steven H. Janzou for help in developing the mesh refinement for the drift-diffusion calculation and Dr. Tom Oppe at Forida State University for setting up the NSPCG solver routines. The authors would also like to thank Drs. Mahmoud Bahrami and Paul Vella of the Digital Equipment Corporation for many useful discussions during the course of this work.

\section{REFERENCES}

(1) S. Y. Chou and M. Y. Liu, "Nanoscale tera-herz metal-semiconductormetal photodetectors," IEEE J. Quant. Electron., vol. 28, pp. 2358-2368, Oct. 1992

[2] L. Figueras and C. W. Slayman, "A novel heterostructure interdigitated photodetector (HIP) with picosecond optical response," IEEE Electron. Dev. Letr., vol. EDL-2, pp. 208-210, Aug. 1981

[3] G. Baccarani, M. Rudan, R. Guerrieri, and P. Ciampolini, "Physical model for numerical device simulation," in Process and Device Madel. ing. W. L. Engl, Ed. Amsterdam: North Holland, 1986, pp. 107-158.

[4] S. Selbether, Analysis and Simulation of Semiconductor Devices. Wien. New York: Springer-Verlag, 1984.

[5] E. Sano, $\leadsto$ device for metal-semiconductor-metal photodetectors and its applications to optoelectronic integrated circuit simulation," IEEE Trans. Electron Devices, vol. 37, pp. 1964-1968, Sept. 1990.

[6] J. E. Sutherland and J. R. Hauser, "A computer analysis of heterojunction and graded composition colar cells," IEEE Trans. Elecrron Devices, vol. ED.24, pp. 363-372. Apr. 1977.

[7] C. M. Wu and E. S. Yang, "Carrier transpon across heterojunction interfaces," Solid State Electron., vol. 22, pp. 241-248, 1979.

[8] K. Horio and H. Yanai, "Numerical modeling of heterojunctions including the thermionic emission mechanism at the heterojunction interface." IEEE Trans. Electron Devices, vol. 37. pp. 1093-98, Apr. 1990.

[9] C. R. Crowell and V. L. Rideout, "Normalized thermionic-field emission in metal-semiconductor (Schotuky) barriers," Solid-Stare Electron., vol. 12. pp. 89-105, 1969.

[10] K. Yang, J. R. East, and G. Haddad, "Numerical modeling of abnupt heterojunctions using a thermionic-field emission boundary condition," Solid State Electron., vol. 36, pp. 321-330, 1993.

[11] C. R. Crowell and S. M. Sze, "Current transport in metal-semiconductor burriers," Solid State Electron., vol. 9. pp. 1035-1048, 1966.

[12] W. L. Engl, H. K. Dirks, and B. Meinerzhagen, "Device modeling," Proc. IEEE, vol. 71, pp. 10-33, Jan. 1983.

[13] D. L. Scharfeter and H. K. Gummel, "Lorge-signal analysis of a silicon read diode oscillator," IEEE Tran. Electron Devices, vol. ED-16, Pp. 64-77. Jan. 1969

[14] G. H. Golub and C. F. Van Loan, Marrix Computations. Baltimore: Johns Hopkins Univ. Press, pp. 362-379, 1983.

[15] T. C. Oppe, W. D. Jouber, and D. R. Kincaid, "An overview of NSPCO ansymmetric preconditioned conjugate gradient package," Compuser Physics Commun., vol. 53, pp. 283-293, 1989.

(16) D. Lendheet, Z-M. Li, S. P. McAlister, and D. A. Aruliah, "Modeling of ultrafast metal-semiconductor-metal photodelectors," Can. J. Phys., vol. $69,1991$.

[17] P. A. Basore, D. T. Rover, and A. W. Smith, "PC-1D version 2; enhanced numerical simulation," Proc. 20ith IEEE Phorovolsaic Spec. Conf., IEEE, New York, 1988, p. 389.
[18] A. W. Smith, "Light confinement and hydrodynamic modelling of ecmiconductor ectuctures by volumetric methods," Ph.D. dissertation, Georgia Inst. of Technol., Atlenta, GA, 1992.

[19] H. C. Casey, Jr. and M. B. Panish, Helerostucture Lasers. New York: Academic, 1978.

[20] K. Horio and H. Yani, "Numerical modeling of heterojunctions including the thermionic emission mechanism at the heterojunction interface," IEEE Trans. Electron Devices, vol. 37, pp. 1093-1098, Apr. 1990.

121] M. Bahremi, private communication, Digita Equipment Corp.

[22] S. A. Ringel, "Growth and process induced defects and recombination mechanisms in AGAASGaAs and CAZnTe/CdS photovoltaic device tructures," Ph.D. disserntion, Georgia Inst of Technol., Allante, GA, 1992.

[23] S. M. Sze, Physics of Semiconducsor Devices. New York: Wiley, 1981, p. 750.

[24] $M$. Ito and $O$ Wadn, "Low dart currem GaAs metal-serniconductor-metal (MSM) photodiodes using WSi ${ }_{x}$ contacts." IEEE J. Qmant. Electron., vol. QE-22. pp. 1073-1077, 1986.

[25] D. S. Malhi, J. M. Xu, F. Hegmann, B. Takataki, and R. Surridge, "Effects of material modification on dark current of GaAs MSM photodetectors," 6th Conf an Semi-insularing III-V Materials, 1990. pp. 451-456, Toronto, Canada.

[26] A. Aboudou, J. P. Viloo, D. Decouler, A. Chenouf, E. Delhaye, P. Boissenol, C. Varin. F. Deachamps, and I. Lecuru, "Ultra-low dark current GaAlAsGaAs MSM photodetoctor," Electronics Lets., vol. 27. no. 10, pp. 793-95, May 1991

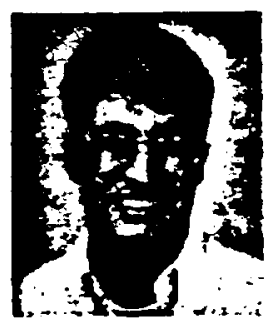

All F. Salem (S'SO) was bom in Marabon, Lebanon. on September 2. 1967. He received the B.S. degree from Boston University, Boston, MA, in 1989 and the M.S. degree from the Georgia Institute of Technology, Allenta, in 1990, both in electrical engineering. He is currently pursuing his Ph.D. degroe in electrical engineering at the Georgia Instiwhe of Technology. His dissertation work involves modeling and simulation of carrier transpon in semiconductor devices and its applications.

Arlynn W. Smith received the B.S. degree in ceramic engineering from Alfred Unviersity in 1984, the M.S. degree from Georgia Institute of Technology in 1987 and the Ph.D. degree in electrical engineering from the Georgia Institute of Technology, Aulante, GA, in 1992. From 1992 to 1993 he held - post-doctoral fellowship at the Georgia Tech Research Institute, where the developed a three dimensional simulation code for modelling the chemical vapor infiltration of ceramic matrix composites. Currently, he is performing a post-doctonl research progrum with the microelectronics research center at the Georgia lnstitute of Technology developing a hydrodynamic simulation code of heterojunction acoustic charge transport devices, charge transpon devices, and avalanche photodiodes.

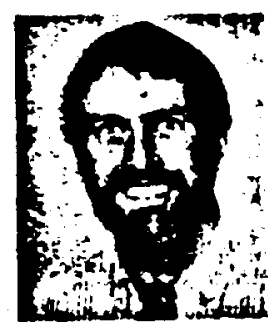

Kevin Breanan (S'84-M'84-SM'90) received the B.S. degree in physics from the Massechusetts ln stitute of Technology, Cembridge, MA, in 1978. and the M.S. degreses in physics in 1980, and the Ph.D. degree in electrical engineering from the Univernity of llinois, Urban-Chempeign, II, in 1984.

He is currently a Professor of Electrical and Computer Engineering at the Georgia Institute of Tochnology, Allanth, GA. His current research interests include the theory and modeling of avalanche devices and confined state photomultipliers, the physics of impact ionization, nonlinear transpon effects in temiconductors and heterostructures, plasmons in eemiconductors, and the theory and modeling of acoustic charge transport devices.

Dr. Brennan was awarded a Presidentia Young Investigator Awand through the National Science Foundation in 1988. 